\title{
Temperature dependence of the current-induced domain wall motion from a modified Landau-Lifshitz-Bloch equation
}

\author{
C. Schieback, D. Hinzke, M. Kläui, U. Nowak, * and P. Nielaba \\ Fachbereich Physik, Universität Konstanz, Universitätsstraße 10, 78457 Konstanz, Germany
}

\begin{abstract}
We employ the Landau-Lifshitz-Bloch (LLB) equation to investigate current-induced domain wall motion at finite temperatures by numerical micromagnetic simulations. We extend the LLB equation with spin torque terms that account for the effect of spin-polarized currents and we find that the velocities depend strongly on the interplay between adiabatic and nonadiabatic spin torque terms. As a function of temperature, we find nonmonotonous behavior, which might be useful to determine the relative strengths of the spin torque terms experimentally.
\end{abstract}

\section{INTRODUCTION}

Magnetic nanostructures in external magnetic fields as well as under the influence of spin-polarized currents have become interesting research fields in recent years due to fundamental novel effects that occur for geometrically confined spin structures, such as domain walls. ${ }^{1}$ Current-induced domain wall motion has been suggested as an alternative to the use of external magnetic fields to induce switching, opening the possibility of simple device fabrication making fieldgenerating strip lines redundant. While current-induced domain wall motion is experimentally well established, ${ }^{2,3}$ the underlying physical mechanisms are not completely understood yet and, in particular, the importance of the adiabatic and the nonadiabatic spin torque terms as well as domain wall transformations for high current densities are highly debated. ${ }^{4-6}$ Furthermore, the influence of temperature on the effects has so far been neglected in the $0 \mathrm{~K}$ calculations and so the experimentally found temperature dependence of, for instance, the critical current densities is so far not understood. $^{7}$

To theoretically predict the behavior of a spin texture under current, one can numerically solve the Landau-LifshitzGilbert (LLG) equation and computer simulations can be performed using either a micromagnetic model or a classical atomistic spin model. Spin torque effects can be taken into account by including the adiabatic and the nonadiabatic torque terms. ${ }^{5,6,8,9}$ Due to the computational expense of atomistic simulations, system sizes are restricted to a nanometer range so that micromagnetic approaches are desirable. However, conventional micromagnetic calculations for larger system sizes lack the correct description of temperature effects because of the assumption of a constant magnetization length. An alternative approach that has only recently started to be used widely to investigate realistic systems sizes including temperature effects is to employ the so-called Landau-Lifshitz-Bloch (LLB) equation. ${ }^{10}$ This equation forms the basis for micromagnetic calculations at elevated temperatures using a macrospin model where longitudinal relaxation processes are taken into account ${ }^{11}$ but so far the LLB equation has only been studied without the spin torque terms.

In this paper, we extend the LLB equation of motion by adding the spin torque terms and we study domain wall mo- tion under the influence of current and at variable temperatures. We determine the domain wall velocities and find that they exhibit a strong dependence on the temperature. Furthermore, by the interplay between the adiabatic and the nonadiabatic spin torque the resulting onset of domain wall transformations (Walker breakdown) is very sensitive to the temperature.

\section{MODEL}

\section{A. Landau-Lifshitz-Bloch equation}

While in the LLG equation at $0 \mathrm{~K}$ the length of the macrospins stays constant, for finite temperatures an equation of motion for macrospins allowing for longitudinal relaxation was derived by Garanin ${ }^{10}$ within mean-field approximation from the classical Fokker-Planck equation for atomistic spins interacting with a heat bath. The resulting "Landau-LifshitzBloch equation" has been shown to be able to describe linear domain walls, a domain wall type with nonconstant magnetization length. ${ }^{12-14}$ Furthermore, the predictions for the longitudinal and transverse relaxation times have been successfully compared with atomistic simulations ${ }^{15}$ as well as rapid heating experiments. ${ }^{16}$ Therefore, we now employ this equation to study the thermodynamics as well as the excitations of macrospins due to currents.

The LLB equation can be written in the form

$\dot{\vec{m}}_{i}=-\gamma \vec{m}_{i} \times \vec{H}_{\mathrm{eff}}^{i}-\frac{\gamma \alpha_{\perp}}{m_{i}^{2}} \vec{m}_{i} \times\left(\vec{m}_{i} \times \vec{H}_{\mathrm{eff}}^{i}\right)+\frac{\gamma \alpha_{\|}}{m_{i}^{2}}\left(\vec{m}_{i} \cdot \vec{H}_{\mathrm{eff}}^{i}\right) \vec{m}_{i}$,

where $\vec{m}_{i}$ is the spin polarization normalized to its zerotemperature value and $\gamma$ the gyromagnetic ratio. The magnetization is not assumed to be of constant length and even its equilibrium value, $m_{\mathrm{e}}$, is temperature dependent. Hence, besides the usual precession and relaxation terms, the LLB equation contains another term which controls longitudinal relaxation.

The LLB equation is valid for finite temperatures and even above the Curie temperature $T_{\mathrm{C}}$ though the damping parameters and effective fields are different below and above $T_{\mathrm{C}} \cdot \alpha_{\|}$and $\alpha_{\perp}$ are dimensionless longitudinal and transverse 
damping parameters. For $T \leq T_{\mathrm{C}}$ they are $\alpha_{\|}=2 \lambda T /\left(3 T_{\mathrm{C}}\right)$ and $\alpha_{\perp}=\lambda\left[1-T /\left(3 T_{\mathrm{C}}\right)\right]$. For $T \geq T_{\mathrm{C}}$ the damping parameters are equal, $\alpha_{\perp}=\alpha_{\|}=2 \lambda T /\left(3 T_{\mathrm{C}}\right)$. Here, $\lambda$ is a microscopic damping parameter which characterizes the coupling of the individual, atomistic spins to the heat bath. In the limit $T \rightarrow 0$ the longitudinal damping parameter $\alpha_{\|}$vanishes and with $\alpha_{\perp}=\lambda$ the LLB equation evolves into the usual LandauLifshitz (LL) equation.

The effective fields of the LLB equation are the derivative $\vec{H}_{\mathrm{eff}}^{i}=-\frac{1}{M_{\mathrm{s}}^{0}} \frac{\delta f}{\delta \vec{m}_{i}}$ of the free-energy density $f$. The total field is given by ${ }^{10}$

$$
\vec{H}_{\mathrm{eff}}^{i}=\vec{H}_{\mathrm{A}}^{i}+\vec{H}_{\mathrm{ex}}^{i}+ \begin{cases}\frac{1}{2 \widetilde{\chi}_{\|}}\left(1-\frac{m_{i}^{2}}{m_{\mathrm{e}}^{2}}\right) \vec{m}_{i} & T \leq T_{\mathrm{C}} \\ -\frac{1}{\widetilde{\chi}_{\|}}\left[1+\frac{2}{5} \frac{T_{\mathrm{C}} m_{i}^{2}}{\left(T-T_{\mathrm{C}}\right)}\right] \vec{m}_{i} & T \geq T_{\mathrm{C}}\end{cases}
$$

with the biaxial anisotropy field,

$$
\vec{H}_{\mathrm{A}}^{i}=-\frac{1}{\tilde{\chi}_{\perp}}\left(\frac{1}{2} m_{y}^{i} \vec{e}_{y}+m_{z}^{i} \vec{e}_{z}\right),
$$

which makes the $x$ axis the easy axis, the $y$ axis the intermediate axis, and the $z$ axis the hard axis of the model. The exchange field is

$$
\vec{H}_{\mathrm{ex}}^{i}=\frac{2 A}{m_{\mathrm{e}}^{2} M_{\mathrm{s}}^{0} \Lambda^{2}} \sum_{j}\left(\vec{m}_{j}-\vec{m}_{i}\right),
$$

where $\Lambda$ is the lateral size of the discretized cells, $A$ is the temperature-dependent exchange stiffness, and $M_{\mathrm{s}}^{0}$ is the zero-temperature saturation magnetization. The susceptibilities $\tilde{\chi}_{l}$ are defined by $\tilde{\chi}_{l}=\partial m_{l} / \partial B_{l}$ with $l=\|, \perp$. Note that at low temperatures the perpendicular susceptibility $\tilde{\chi}_{\perp}$ is related to the temperature-dependent anisotropy constant $K$ via $\tilde{\chi}_{\perp}=M_{\mathrm{s}}^{0} m_{\mathrm{e}}^{2} /(2 K) .{ }^{10}$ We use functions for $\widetilde{\chi}_{l}(T), m_{\mathrm{e}}(T)$, and $A(T)$ as calculated before for the spin model (for details see Refs. 11 and 14) but rescaled to reflect a ferromagnetic material with a Curie temperature of $1043 \mathrm{~K}$ and $M_{\mathrm{s}}^{0}$ of $10^{6} \mathrm{~A} / \mathrm{m}$. Furthermore, we normalize the perpendicular susceptibility such that its value at $0 \mathrm{~K}, \tilde{\chi}_{\perp}(T=0)=M_{\mathrm{s}}^{0} / 2 K_{\mathrm{x}}$ corresponds to an anisotropy of $K_{\mathrm{x}}=10^{5} \mathrm{~J} / \mathrm{m}^{3}$ and $2 K_{\mathrm{y}}=K_{\mathrm{x}}$. These functions are shown in Figs. 1 and 2.

\section{B. Spin torque in the Landau-Lifshitz-Bloch equation}

Throughout this paper we will consider a one-dimensional model of a domain wall. An established approach for the effect of a spin-polarized current in the $x$ direction on a domain wall is presented in Ref. 6. In these studies the interaction between electron spins and magnetization has been treated by additional spin torque terms

$$
\vec{T}=-u_{\mathrm{x}} \frac{\partial \vec{S}}{\partial x}+\beta \vec{S} \times u_{\mathrm{x}} \frac{\partial \vec{S}}{\partial x},
$$

where $\vec{S}$ is a unit vector representing the direction of the magnetization. The first contribution to the spin torque is

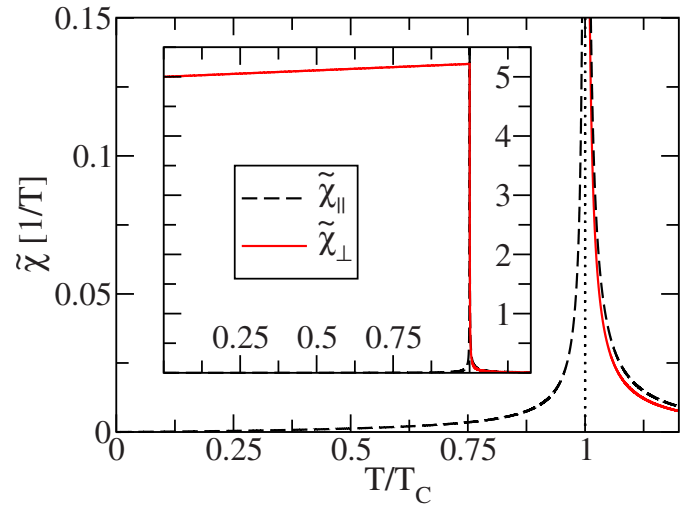

FIG. 1. (Color online) Equilibrium parallel and transverse susceptibility vs temperature determined as explained in the text. The inset shows the transverse susceptibility vs temperature on a larger scale.

called the adiabatic term. It can be derived from an additional term in the magnetic free energy that takes into account the coupling of the magnetization to the spins of the electrical current, ${ }^{17}$ representing an adiabatic transfer of angular momentum to the magnetization. In the adiabatic limit the spin polarization of the current is always oriented along the local direction of the magnetization. The second contribution is the nonadiabatic term that reflects the mistracking of the direction of the conduction-electron spins with respect to the magnetization. It appears to play a role similar to the Gilbert damping term. Recent micromagnetic numerical investigations ${ }^{5,6,18-20}$ using a modified LLG equation including these terms have given a qualitative insight into the roles played by these two torque terms.

In the case of the current flowing in the $x$ direction, the magnitude of the effective spin current $u_{\mathrm{x}}$ is given by $u_{\mathrm{x}}=j_{\mathrm{x}} / M$, where $M$ is the magnetization and $j_{\mathrm{x}}$ the spin current density $j_{\mathrm{x}}=\mu_{\mathrm{B}} P j_{\mathrm{e}} / e$ which is proportional to the electrical current density $j_{\mathrm{e}}$ and to the polarization $P$. Here, $\mu_{\mathrm{B}}$ is the Bohr magneton and $e$ the magnitude of the electron charge. So far, the polarization $P$ and thus the resulting spin current density $j_{\mathrm{x}}$ have been assumed to be temperature independent. In the following, we extend the model so that a

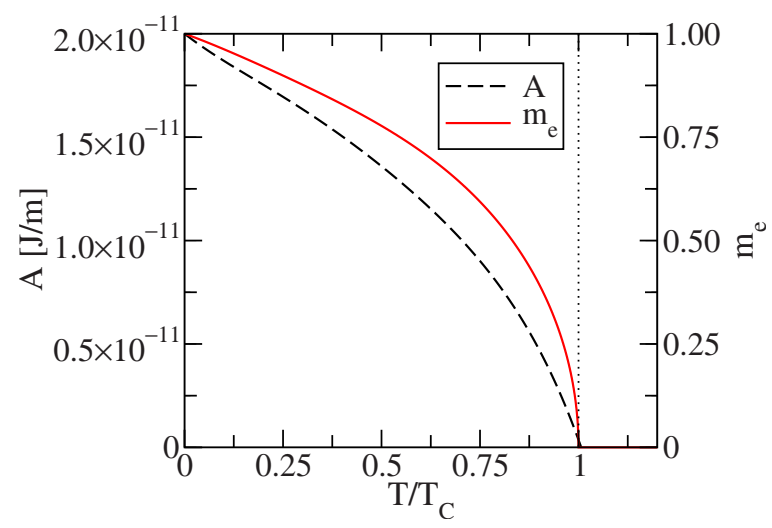

FIG. 2. (Color online) Exchange stiffness $A$ and reduced equilibrium magnetization $m_{\mathrm{e}}$ vs temperature determined as explained in the text. 
temperature-dependent spin current $j_{\mathrm{x}}(T)$ is taken into account. Under the assumption that the spin polarization is proportional to the magnetization, $P(M)=P^{0} m$, and with $M=M_{\mathrm{s}}^{0} m, u_{\mathrm{x}}$ is $u_{\mathrm{x}}=\frac{P^{0} j_{\mathrm{e}} \mu_{\mathrm{B}}}{M_{\mathrm{s}}^{0} e}$ for all temperatures.

Under these assumptions the spin torque terms can be expressed in terms of the reduced magnetization $m$ as

$$
\vec{T}=-u_{\mathrm{x}} \frac{\partial \vec{m}}{\partial x}+\frac{\beta}{m} \vec{m} \times u_{\mathrm{x}} \frac{\partial \vec{m}}{\partial x} .
$$

Note that the spin torque now is temperature dependent via the variable $m$ which, within the framework of the LLB equation, is no longer a unit vector of constant length. For the same reason, the adiabatic torque term can no longer be expressed as a double cross product. Instead it is

$$
-u_{\mathrm{x}} \frac{\partial \vec{m}}{\partial x}=\frac{u_{\mathrm{x}}}{m^{2}}\left[\vec{m} \times\left(\vec{m} \times \frac{\partial \vec{m}}{\partial x}\right)-\vec{m} \cdot\left(\vec{m} \cdot \frac{\partial \vec{m}}{\partial x}\right)\right],
$$

which means that the adiabatic term, within the LLB equation, gives rise to an additional longitudinal spin torque term which vanishes in the LLG equation due to the assumption of a constant length of the magnetization vector. As pointed out in Ref. 21 this term corresponds to the spin accumulation and in the metal systems considered here, it constitutes usually a small effect.

It is not yet clear whether the spin torque term $\vec{T}$ should be added to the Landau-Lifshitz or the Landau-Lifshitz-Gilbert form of the equation of motion (for more details see the discussion in Refs. 22-24). The same problem arises with the LLB equation. In the following, we extend the LLB equation with both forms of the damping, the one after Landau and Lifshitz as well as the one after Gilbert. The LL form of the LLB equation [Eq. (1)] is the original one as derived by Garanin. ${ }^{10}$ Equation (1) now reads with the additional spin torque terms from Eq. (6)

$$
\begin{aligned}
\dot{\vec{m}}_{i}= & -\gamma \vec{m}_{i} \times \vec{H}_{\mathrm{eff}}^{i}-\frac{\gamma \alpha_{\perp}}{m_{i}^{2}} \vec{m}_{i} \times\left(\vec{m}_{i} \times \vec{H}_{\mathrm{eff}}^{i}\right) \\
& +\frac{\gamma \alpha_{\|}}{m_{i}^{2}}\left(\vec{m}_{i} \cdot \vec{H}_{\mathrm{eff}}^{i}\right) \vec{m}_{i}-u_{\mathrm{x}} \frac{\partial \vec{m}_{i}}{\partial x}+\frac{\beta_{\mathrm{LL}}}{m_{i}} \vec{m}_{i} \times u_{\mathrm{x}} \frac{\partial \vec{m}_{i}}{\partial x} .
\end{aligned}
$$

Neglecting terms of the order of $\alpha^{2}$ the LLB equation can be transformed into an equation with a damping term following Gilbert. Adding the same spin torque terms $\vec{T}$ to this form of the LLB equation yields

$$
\begin{aligned}
\dot{\vec{m}}_{i}= & -\gamma \vec{m}_{i} \times \vec{H}_{\mathrm{eff}}^{i}+\frac{\gamma \alpha_{\perp}}{m_{i}^{2}} \vec{m}_{i} \times \dot{\vec{m}}_{i}+\frac{\gamma \alpha_{\|}}{m_{i}^{2}}\left(\vec{m}_{i} \cdot \vec{H}_{\mathrm{eff}}^{i}\right) \vec{m}_{i} \\
& -u_{\mathrm{x}} \frac{\partial \vec{m}_{i}}{\partial x}+\frac{\beta_{\mathrm{G}}}{m_{i}} \vec{m}_{i} \times u_{\mathrm{x}} \frac{\partial \vec{m}_{i}}{\partial x} .
\end{aligned}
$$

Note that we use the notation $\beta_{\mathrm{G}}$ and $\beta_{\mathrm{LL}}$ for the nonadiabatic prefactor only for convenience in order to distinguish in the following between the LL and LLG form of the LLB equation.

In the next step we transform Eq. (9) into an explicit form so that we are able to compare it with Eq. (8) and also since an explicit equation is more convenient for a numerical treat- ment. This explicit equation can be derived once again neglecting terms of the order of $\alpha^{2}$ and $\alpha \beta$ and it is given by

$$
\begin{aligned}
\dot{\vec{m}}_{i}= & -\gamma \vec{m}_{i} \times \vec{H}_{\mathrm{eff}}^{i}-\frac{\gamma \alpha_{\perp}}{m_{i}^{2}} \vec{m}_{i} \times\left(\vec{m}_{i} \times \vec{H}_{\mathrm{eff}}^{i}\right) \\
& +\frac{\gamma \alpha_{\|}}{m_{i}^{2}}\left(\vec{m}_{i} \cdot \vec{H}_{\mathrm{eff}}^{i}\right) \vec{m}_{i}-u_{\mathrm{x}} \frac{\partial \vec{m}_{i}}{\partial x}+\left(\frac{\beta_{\mathrm{G}}}{m_{i}}-\frac{\alpha_{\perp}}{m_{i}^{2}}\right) \vec{m}_{i} \times u_{\mathrm{x}} \frac{\partial \vec{m}_{i}}{\partial x} .
\end{aligned}
$$

The only difference between Eq. (8) assuming LandauLifshitz damping, and Eq. (10) assuming Gilbert damping, is the prefactor of the last term. Equations (8) and (10) are mathematically identical for $\beta_{\mathrm{LL}}=\beta_{\mathrm{G}}-\alpha_{\perp} / m_{i}$. Note that at 0 $\mathrm{K}$ both equations evolve into the well-established explicit versions of the LL, respectively, LLG equation with spin torque terms. ${ }^{22-24}$

\section{ANALYTICAL MODEL}

In the following, we present analytical calculations for a one-dimensional system aligned along the $x$ direction. We extended well established, analytical $0 \mathrm{~K}$ calculations ${ }^{6,18}$ to elevated temperatures using the LLB equation as equation of motion. Note that within the LLB approach domain wall profiles change with increasing temperature first from circular to elliptical and later on to linear. ${ }^{12-14}$ However, these effects occur only at higher temperatures close to $T_{\mathrm{C}}$ (depending on the strength of the anisotropy) and are neglected in our analytical calculations. Hence, we assume a temperatureindependent domain wall type, which here is a transverse domain wall along the $x$ direction, which does not change its spin structure dynamically. This assumption is later tested by comparison with numerical calculations without these approximations.

In Refs. 13 and 14 it was shown that at finite temperatures the mathematical form of a transverse wall profile (e.g., Bloch type), which can be described by the usual hyperbolic functions, is conserved; solely the amplitude and the domain wall width vary with temperature. The assumed transverse domain wall profile is

$$
\vec{m}=-m_{\mathrm{e}}\left\{\tanh \left(\frac{x}{\Delta}\right) \vec{e}_{x}+\frac{\cos (\phi)}{\cosh \left(\frac{x}{\Delta}\right)} \vec{e}_{y}+\frac{\sin (\phi)}{\cosh \left(\frac{x}{\Delta}\right)} \vec{e}_{z}\right\}
$$

with the temperature-dependent domain wall width

$$
\Delta(T, \phi)=\sqrt{\frac{4}{M_{\mathrm{s}}^{0}} \frac{A(T) \tilde{\chi}_{\perp}(T)}{m_{\mathrm{e}}^{2}(T)} \frac{1}{\left(1+\sin ^{2} \phi\right)}}
$$

and the out-of-plane angle $\phi$. In the low-temperature limit this equation has the well-known form, $\Delta=\sqrt{2 A /\left[K\left(1+\sin ^{2} \phi\right)\right]}$.

The equations of motion are calculated as described in Ref. 9. We assume Gilbert damping first, simply because it is the more common assumption in connection with spin torque calculations. For the domain wall profile above the equations 


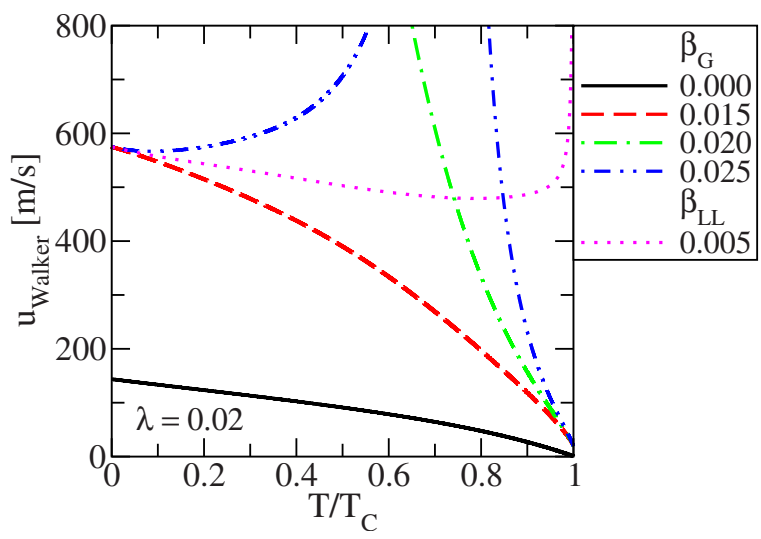

FIG. 3. (Color online) Walker threshold $u_{\text {Walker }}$ according to Eq. (15) vs the reduced temperature $T / T_{\mathrm{C}}$ for different values of the nonadiabatic prefactor $\beta_{\mathrm{G}}$ as well as $\beta_{\mathrm{LL}}$.

of motion for the position of the domain wall $x_{\mathrm{G}}$ and the angle $\phi$ are

$$
\begin{gathered}
\dot{x}_{\mathrm{G}}=\frac{m_{\mathrm{e}} \Delta \gamma}{4 \tilde{\chi}_{\perp}} \sin 2 \phi+u_{\mathrm{x}}, \\
\dot{\phi}=-\frac{\alpha_{\perp} \gamma}{4 \widetilde{\chi}_{\perp}} \sin 2 \phi-\frac{\left(\alpha_{\perp}-\beta_{\mathrm{G}} m_{\mathrm{e}}\right)}{m_{\mathrm{e}}} \frac{u_{\mathrm{x}}}{\Delta} .
\end{gathered}
$$

These equations are calculated based on the original procedure (see Ref. 6 and references therein). The corresponding Walker threshold $u_{\text {Walker }}^{\mathrm{G}}$ (Ref. 25) can be calculated under the assumptions that $\dot{\phi}=0$ and that the function $\Delta(T, \phi) \sin 2 \phi$ within Eq. (14) reaches its maximum at $\phi \approx \pi / 4$. The Walker threshold is then given by

$$
u_{\text {Walker }}^{\mathrm{G}}=\frac{\gamma}{4 \widetilde{\chi}_{\perp}} \Delta_{\text {Walker }} \frac{\alpha_{\perp} m_{\mathrm{e}}}{\left|\alpha_{\perp}-\beta_{\mathrm{G}} m_{\mathrm{e}}\right|}
$$

with $\Delta_{\text {Walker }}=\Delta(T, \phi=\pi / 4)$ and the average domain wall velocity is

$$
\langle v\rangle_{\mathrm{G}}=\frac{\beta_{\mathrm{G}} u_{\mathrm{x}} m_{\mathrm{e}}}{\alpha_{\perp}} \pm \frac{m_{\mathrm{e}} \Delta_{\text {Walker }} \gamma}{4 \widetilde{\chi}_{\perp}} \sqrt{\left(\frac{u_{\mathrm{x}}}{u_{\text {Walker }}^{\mathrm{G}}}\right)^{2}-1}
$$

with the plus sign for $\left(\alpha_{\perp}-\beta_{\mathrm{G}} m_{\mathrm{e}}\right) u_{\mathrm{x}}>0$ and the minus sign otherwise. This equation contains a contribution which is linear in the current (in the nonadiabatic case) and a second square-root contribution above the Walker threshold. The temperature dependence is included in the temperaturedependent parameters $A(T), m_{\mathrm{e}}(T), \chi_{\perp}(T)$, and $\alpha_{\perp}(T)$. At zero temperature these results are identical to those gained with the LLG equation. ${ }^{6,18}$ The longitudinal spin torque does not affect the analytical results since we assume a constant domain wall type. Very close to the Curie temperature this assumption is no longer valid and deviations can be expected, which are beyond the scope of the current investigation.

In Fig. 3 the Walker threshold according to Eq. (15) is shown as a function of the reduced temperature $\left(T / T_{\mathrm{C}}\right)$. In all cases the Walker threshold vanishes at the Curie temperature. In the adiabatic case $\left(\beta_{\mathrm{G}}=0\right)$ and in general for
$\beta_{\mathrm{G}}<\lambda=\alpha_{\perp}(T=0)$ it decreases with increasing temperatures. For $\beta_{\mathrm{G}}=\lambda=0.02$ the Walker threshold diverges approaching zero temperature. The behavior for $\beta_{\mathrm{G}}>\lambda$ is similar but here the Walker threshold diverges at a finite temperature. As we will see in the following, the understanding of the temperature dependence of the Walker threshold is the key for understanding the temperature dependence of the domain wall velocity.

The equation of motion for Landau-Lifshitz damping [Eq. (10)] can be calculated and solved in the same way as for the Gilbert damping with corresponding results for the Walker threshold and the average domain wall velocity for $\beta_{\mathrm{LL}}=\beta_{\mathrm{G}}-\alpha_{\perp} / m_{\mathrm{e}}$. Note, however, that since this transformation is temperature dependent, the temperature dependence of both, Walker threshold and domain wall velocity, are different for Landau-Lifshitz and Gilbert damping, respectively. The temperature dependence of the Walker threshold assuming Landau-Lifshitz damping is also shown in Fig. 3. The main difference is that approaching the Curie temperature the Walker threshold does not vanish but diverges. In the limit of low temperatures, however, the Walker threshold converges to the one following Gilbert damping.

\section{NUMERICAL MODEL}

By means of computer simulations, temperaturedependent domain wall velocities were calculated for a onedimensional system of $512 \mathrm{~nm}$ length, discretized with $1 \mathrm{~nm}$ cell size. The initial magnetization configuration was a planar domain wall positioned in the middle of the chain with the temperature-dependent profile and width given by Eqs. (11) and (12) with $\phi=0$. For $0 \mathrm{~K}$ the domain wall width varies between $\Delta_{\max }=20 \mathrm{~nm}(\phi=0)$ and $\Delta_{\min }=14.1 \mathrm{~nm}$ $(\phi=\pi / 2)$ with $\Delta_{\text {Walker }}=16.3 \mathrm{~nm}(\phi=\pi / 4)$. At the ends of the system the spins were fixed as boundary conditions in the $x$ direction and $-x$ direction, respectively. To minimize the influence of these boundary conditions the domain wall was only allowed to move within a range of $60 \mathrm{~nm}$ from the center of the system. When the domain wall moved out of this interval, it was shifted back along the $x$ coordinate and repositioned at the opposite side of the interval. The domain wall velocity was calculated from the derivative of the spatially averaged $x$ component of the magnetization versus time. The numerical time integration of Eqs. (8) and (10) was carried out using a Heun method ${ }^{26,27}$ with 1.8 fs time step size.

\section{RESULTS}

\section{A. Adiabatic spin torque effect}

In the following, first the pure adiabatic spin torque effect $\left(\beta_{\mathrm{G}}=0\right)$ is discussed. For zero temperature, this effect was already investigated previously based on the LLG equation. ${ }^{18,19,28,29}$ It was found that for low effective spin currents $u_{\mathrm{x}}$ the domain wall moves along the wire until it reaches a maximum displacement where it stops. At the same time, the magnetization of the domain wall is tilted out of the easy plane up to a maximum out-of-plane angle. This behavior can be explained by an analysis of the different terms of 

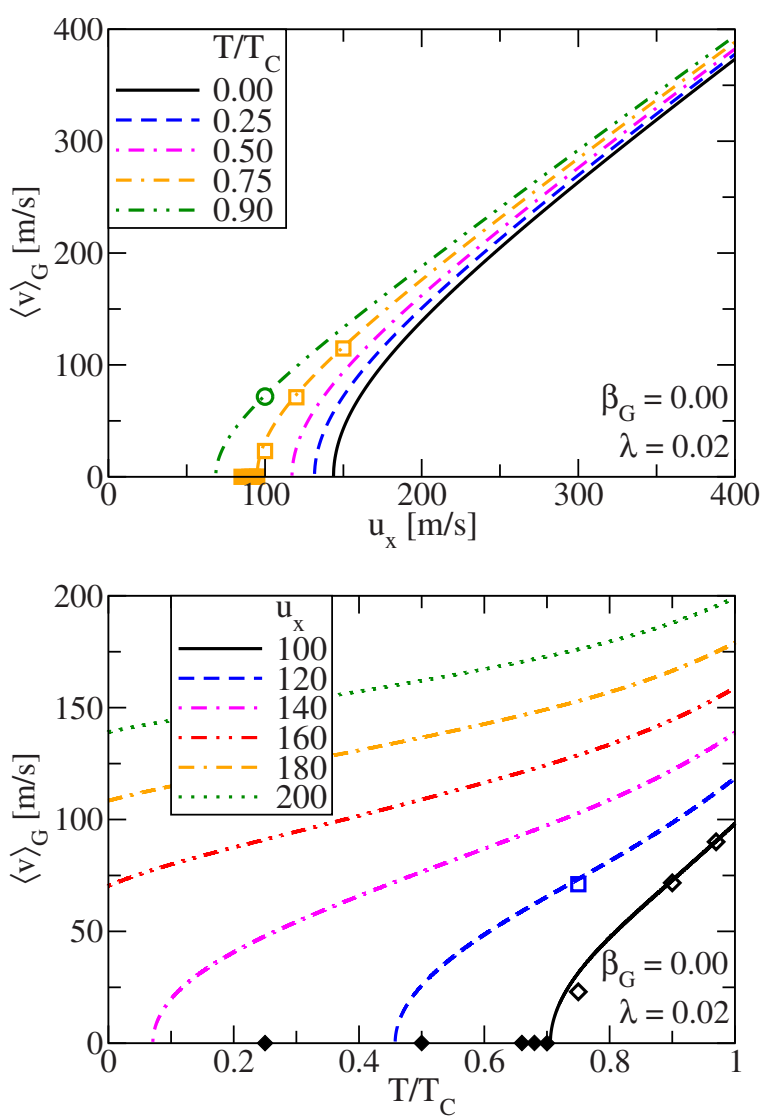

FIG. 4. (Color online) The top figure shows the average domain wall velocity $\langle v\rangle_{\mathrm{G}}$ calculated from the LLB equation with Gilbert damping vs the effective spin current $u_{\mathrm{x}}$ for different temperatures. The solid line for $T=0 \mathrm{~K}$ represents the case where the LLB and the LLG equations are identical. The bottom figure shows $\langle v\rangle_{\mathrm{G}}$ vs the reduced temperature $T / T_{\mathrm{C}}$. The lines represent the analytic solution [Eq. (16)] and the points are from numerical simulations. The calculations are for the adiabatic case, $\beta_{\mathrm{G}}=0$ and $\lambda=0.02$.

the extended LLG equation [Eq. (10)]: the first spin torque term which moves the domain wall is balanced by an "internal" torque due to the anisotropy contribution to the effective field. The displacement of the domain wall in $x$ direction is eventually stopped by the precessional term acting in the opposite direction while the second spin torque term which is responsible for tilting the magnetization out of the easy plane is balanced by the damping term.

It was even analytically predicted ${ }^{18,19,28}$ that the averaged domain wall velocity as a function of the effective spin current remains zero unless the current exceeds a critical value $u_{\mathrm{c}}$. This predicted critical current was also found in atomistic simulations at $0 \mathrm{~K} .{ }^{29}$ Below the critical effective spin current $\left(u_{\mathrm{x}}<u_{\mathrm{c}}\right)$ no continuous domain wall motion is observed while above the critical current the spin torque term can no longer be balanced by the anisotropy. Consequently domain wall motion occurs in addition to a precession of the magnetization around the $x$ axis.

We find the same behavior in the extended LLB equation. This can be seen in Fig. 4 where the averaged domain wall velocity is shown as a function of the effective spin current $u_{\mathrm{x}}$ for different reduced temperatures $T / T_{\mathrm{C}}$. However, it is found that the critical effective spin current is temperature dependent, following the equation

$$
u_{\mathrm{c}}=\frac{\gamma m_{\mathrm{e}}}{4 \tilde{\chi}_{\perp}} \Delta_{\text {Walker }}
$$

[Eq. (15) for $\beta_{\mathrm{G}}=0$ ]. For larger temperatures the critical current decreases since thermodynamically the anisotropy decreases. This leads to the fact that domain walls at higher temperatures are faster than at low temperatures.

Furthermore, in the bottom part of Fig. 4 the averaged domain wall velocity is shown as a function of the reduced temperature for different values of the effective spin current. In the limit of low current a critical temperature $T^{\star}$ can be identified. For $T<T^{\star}$ no continuous domain wall motion is observed while for $T>T^{\star}$ domain wall motion occurs. This critical temperature is shifted to lower values for higher spin currents. In the limit of high effective spin current $T^{\star}$ vanishes and domain wall motion can be observed over the whole temperature range. Here, the spin torque effect is no longer balanced by the anisotropy and only the terms responsible for the precession of the magnetization around the $x$ axis affects the domain wall motion.

In both figures, analytical curves and numerical results agree. This demonstrates clearly that the assumptions made for the derivation of Eq. (16) are reasonable for the parameters used. In particular, it shows that the influence of the longitudinal spin torque on the domain wall motion is unimportant in the range of temperatures under investigation.

Note that the pinning barrier which stops the domain wall motion can be overcome by thermal fluctuations. The role of these fluctuations was investigated by Duine et al. ${ }^{30}$ within the framework of an extended, stochastic LLG equation. These fluctuations are relevant in the limit of very thin wires where by thermal activation the pinning potential can be overcome on sufficiently long time scales, leading to a finite domain wall motion even below the critical current. In our work, however, fluctuations are not considered so that the results are more relevant for thicker wires where thermal fluctuations of the domain wall profile can be neglected.

\section{B. Nonadiabatic spin torque effects}

In the following, nonadiabatic spin torque is taken into account and its effect is discussed in more detail. For comparison with previous investigations, ${ }^{6,29}$ the nonadiabatic prefactor $\beta_{\mathrm{G}}$ is assumed to be temperature independent and is investigated in relation to the temperature-independent microscopic damping constant $\lambda$. Note, however, that in Ref. 31 nonlocal contributions, which are strongly correlated with the domain wall width, are predicted, which are neglected for our wide walls here.

Our results for the case $\beta_{\mathrm{G}}=\lambda$ are shown in Fig. 5. In the zero-temperature limit it is $m=1$ and $\alpha_{\perp}=\lambda$ so that the last term of Eq. (10) vanishes and only the first spin torque term remains finite which is responsible for the displacement of the domain wall along the $x$ axis. The magnetization is, hence, not tilted out of the easy plane and no torque occurs due to the precessional or relaxational part of the LLB equation. This behavior was already observed in previous numeri- 

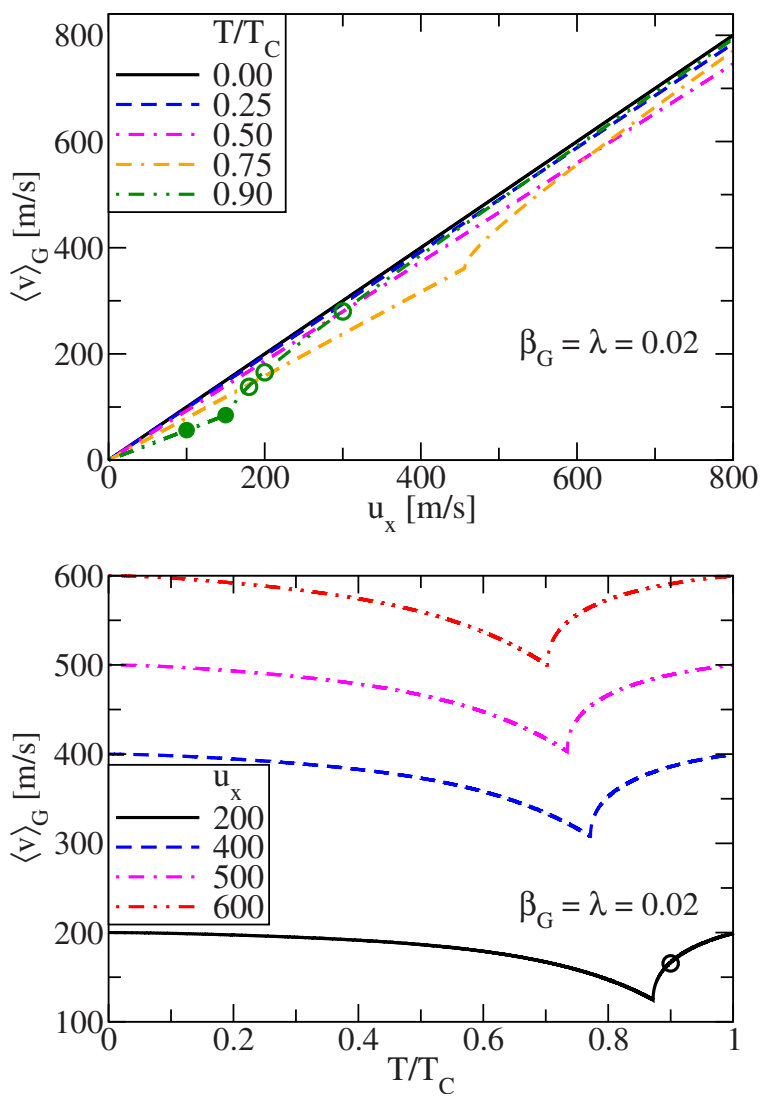

FIG. 5. (Color online) The top figure shows the average domain wall velocity $\langle v\rangle_{\mathrm{G}}$ calculated from the LLB equation with Gilbert damping vs the effective spin current $u_{\mathrm{x}}$ for different temperatures. The bottom figure shows $\langle v\rangle_{\mathrm{G}}$ vs the reduced temperature $T / T_{\mathrm{C}}$. The lines represent the analytic solution [see Eq. (16)] and the points are from numerical simulations. The calculations are for the nonadiabatic case, $\beta_{\mathrm{G}}=\lambda=0.02$.

cal investigations of the LLG equation and is discussed in more detail in Refs. 6 and 29.

In the case of elevated temperatures, the situation is different due to the fact that the last term of Eq. (10) does not vanish because of the temperature dependence of $m$ and $\alpha_{\perp}$. This term is responsible for tilting the magnetization out of the easy plane and it leads to the existence of the Walker threshold $u_{\text {Walker }}^{\mathrm{G}}[\mathrm{Eq} .(15)]$. Figure 5 shows that two regimes can be distinguished: for $u_{\mathrm{x}}<u_{\text {Walker }}^{\mathrm{G}}$ the velocity $\langle v\rangle_{\mathrm{G}}$ shows a linear behavior as in the zero-temperature limit while in the regime $u_{\mathrm{x}}>u_{\text {Walker }}^{\mathrm{G}}$ the second term in Eq. (16) takes over and the velocity increases even faster. Here, the last term of Eq. (10) leads to a continuous rotation of the magnetization around the $x$ axis. Following Eq. (15) the transition between these regimes is shifted to lower effective spin currents with increasing temperature vanishing at $0 \mathrm{~K}$.

The averaged domain wall velocity as a function of temperature for different effective spin current values is shown in the bottom part of Fig. 5. First, it decreases with increasing temperature until a minimum value is reached, after which the velocity increases. The minimum can be identified once again as the Walker threshold. This behavior is found for all effective velocities although the value of the minimum
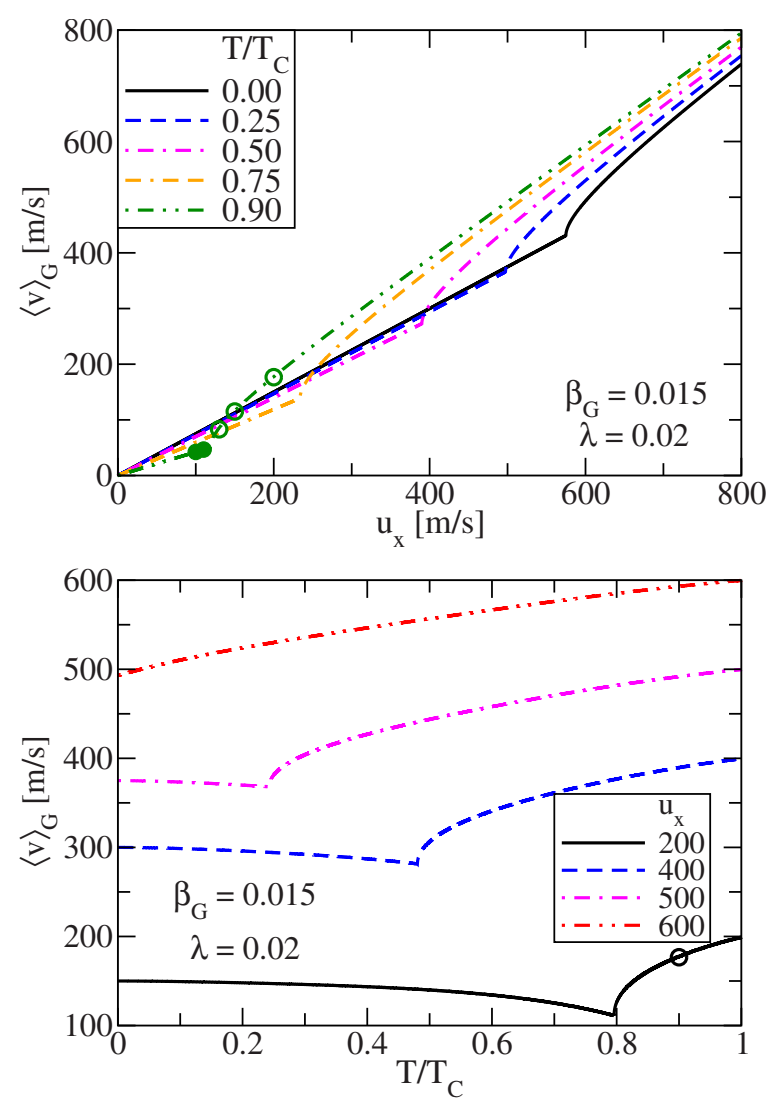

FIG. 6. (Color online) The top figure shows the average domain wall velocity $\langle v\rangle_{\mathrm{G}}$ calculated from the LLB equation with Gilbert damping vs the effective spin current $u_{\mathrm{x}}$ for different temperatures. The bottom figure shows $\langle v\rangle_{\mathrm{G}}$ vs the reduced temperature $T / T_{\mathrm{C}}$. The lines represent the analytic solution [see Eq. (16)] and the points are from numerical simulations. The calculations are for the nonadiabatic case, $\beta_{\mathrm{G}}<\lambda$.

of the velocity is shifted to higher temperatures for lower effective velocities.

Figure 6 shows results for the case $\beta_{\mathrm{G}}<\lambda$. Here, the term responsible for tilting the magnetization out of the easy plane plays a crucial role for all temperatures even at $0 \mathrm{~K}$. As before, the Walker threshold is shifted to lower effective velocities with increasing temperature. In comparison to the case $\beta_{\mathrm{G}}=\lambda$, the Walker threshold occurs at lower effective spin currents for the same temperature value so that the precession of the domain wall sets in earlier.

The averaged domain wall velocity as a function of the temperature is shown in the bottom part of Fig. 6 for different values of the effective spin current. As before a minimum exists which is shifted to lower temperatures for higher effective velocities, consistent with the shift of the Walker threshold discussed above. In comparison to the $\beta_{\mathrm{G}}=\lambda$ case, this shift of the minima is more pronounced.

Finally, Fig. 7 shows results for the case $\beta_{\mathrm{G}}>\lambda$. Here, the behavior differs from the two cases before. First of all, above the Walker threshold, the wall velocity increases slower than linear not faster as before. This is due to the sign change in Eq. (16). Also, starting from low temperature the Walker threshold is first shifted to higher currents. In the effective spin current range shown in the figure the Walker threshold 

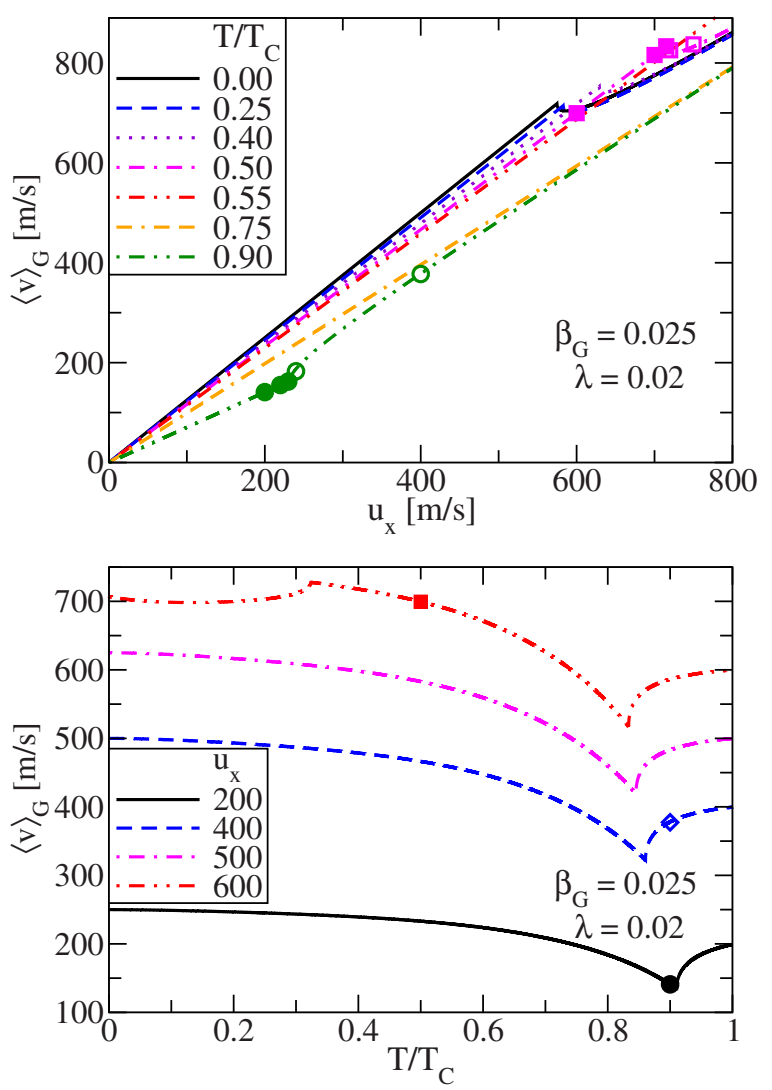

FIG. 7. (Color online) The top figure shows the average domain wall velocity $\langle v\rangle_{\mathrm{G}}$ calculated from the LLB equation with Gilbert damping vs the effective spin current $u_{x}$ for different temperatures. The bottom figure shows $\langle v\rangle_{\mathrm{G}}$ vs the reduced temperature $T / T_{\mathrm{C}}$. The lines represent the analytic solution [see Eq. (16)] and the points are from numerical simulations. For $u_{\mathrm{x}}=600 \mathrm{~m} / \mathrm{s}$ two crossings of the Walker threshold are visible $\left(T / T_{\mathrm{C}}=0.32\right.$ and $\left.T / T_{\mathrm{C}}=0.83\right)$. The calculations are for the nonadiabatic case, $\beta_{\mathrm{G}}>\lambda$.

even disappears due to the fact that it is shifted out of the range presented. Surprisingly, at temperatures close to $T_{\mathrm{C}}$ another Walker threshold appears at lower effective velocities above which the averaged wall velocity increases faster than linear.

This second transition can also be identified from the averaged velocity as a function of temperature as shown in the bottom part of Fig. 7. For an effective spin current of 600 $\mathrm{m} / \mathrm{s}$ there are two temperatures where the behavior of the velocity changes. This corresponds to the fact that the Walker threshold as shown in Fig. 3 can be crossed twice for $\beta_{\mathrm{G}}>\lambda$ and certain values of the effective current, leading to this intricate behavior, which could be easily identified if observed experimentally.

\section{Comparison of Gilbert and Landau-Lifshitz damping}

In this section the difference between the assumption of Gilbert damping on the one hand and Landau-Lifshitz damping on the other hand is discussed for the case $\beta_{\mathrm{G}}>\lambda$. As mentioned before the prefactors for the nonadiabatic spin torque term can be transformed as $\beta_{\mathrm{LL}}=\beta_{\mathrm{G}}-\alpha_{\perp} / m_{\mathrm{e}}$. This
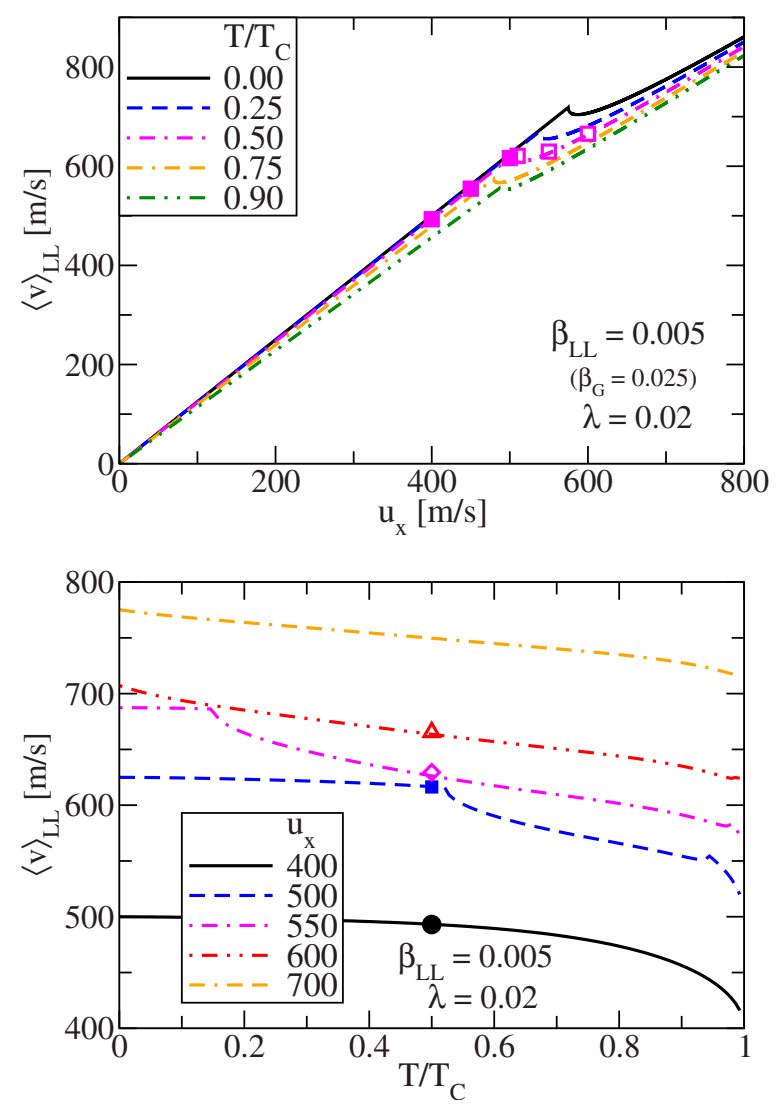

FIG. 8. (Color online) The top figure shows the average domain wall velocity $\langle v\rangle_{\mathrm{G}}$ calculated from the extended LLB equation with Landau-Lifshitz damping vs the effective spin current $u_{\mathrm{x}}$ for different temperatures. The bottom figure shows $\langle v\rangle_{\mathrm{LL}}$ vs the reduced temperature $T / T_{\mathrm{C}}$. The lines represent analytic solutions and the points are from numerical simulations. The calculations are for the nonadiabatic case, nonadiabatic case $\beta_{\mathrm{LL}}=0.005\left(\beta_{\mathrm{G}}=0.025\right)$ and $\lambda=0.02)$.

transformation is temperature dependent so that a qualitatively different temperature dependence exists for LandauLifshitz damping in comparison to the Gilbert damping discussed before. Figure 8 summarizes our results for the extended LLB equation assuming Landau-Lifshitz damping [Eq. (8)].

Comparing Figs. 7 and 8 it is found that the curves for 0 $\mathrm{K}$ are indeed the same. However, the behavior at elevated temperatures is completely different: a Walker threshold can be identified for all temperatures. Below the threshold the domain wall is moved along the wire with an averaged velocity proportional to the effective spin current. Above the threshold additionally to this movement the magnetization precesses around the $x$ axis so that the velocity of the domain wall is decreasing. The temperature-dependent threshold is shifted to lower effective velocities for higher temperatures. Here, the $0 \mathrm{~K}$ curve is an upper limit for the averaged domain wall velocity.

The behavior of the domain wall velocity as a function of the temperature is less complicated than in the corresponding Gilbert case. For low currents the averaged velocity steadily decreases with increasing temperatures. For slightly larger currents the domain wall moves with a constant velocity un- 
til the Walker threshold is reached. Above this point the velocity is decreasing. Close to the Curie temperature another transition point is reached where the domain wall velocity decreases even faster. Here, the Walker threshold is crossed again (see Fig. 3). A further increase in the effective spin current leads to a shift of the first transition point to lower temperatures and only two regimes can be identified.

\section{CONCLUSIONS}

In summary, we combined the LL form of the LLB equation as well as the Gilbert form of the LLB equation with the adiabatic and nonadiabatic spin torque terms. We investigated analytically as well as numerically domain wall motion at various temperatures for the adiabatic and nonadiabatic cases. The Walker threshold as well as the domain wall velocities show a strong temperature dependence. Furthermore, we found a different behavior for the temperature-dependent

*ulrich.nowak@uni-konstanz.de

${ }^{1}$ M. Kläui, J. Phys.: Condens. Matter 20, 313001 (2008).

${ }^{2}$ A. Yamaguchi, T. Ono, S. Nasu, K. Miyake, K. Mibu, and T. Shinjo, Phys. Rev. Lett. 92, 077205 (2004).

${ }^{3}$ M. Kläui, C. A. F. Vaz, J. A. C. Bland, W. Wernsdorfer, G. Faini, E. Cambril, L. J. Heyderman, F. Nolting, and U. Rüdiger, Phys. Rev. Lett. 94, 106601 (2005).

${ }^{4}$ L. Heyne, M. Kläui, D. Backes, T. A. Moore, S. Krzyk, U. Rüdiger, L. J. Heyderman, A. F. Rodríguez, F. Nolting, T. O. Mentes, M. Á. Niño, A. Locatelli, K. Kirsch, and R. Mattheis, Phys. Rev. Lett. 100, 066603 (2008).

${ }^{5}$ Z. Li and S. Zhang, Phys. Rev. Lett. 92, 207203 (2004).

${ }^{6}$ A. Thiaville, Y. Nakatani, J. Miltat, and N. Suzuki, Europhys. Lett. 69, 990 (2005).

${ }^{7}$ M. Laufenberg, W. Bührer, D. Bedau, P.-E. Melchy, M. Kläui, L. Vila, G. Faini, C. A. F. Vaz, J. A. C. Bland, and U. Rüdiger, Phys. Rev. Lett. 97, 046602 (2006).

${ }^{8}$ L. Berger, J. Appl. Phys. 49, 2156 (1978).

${ }^{9}$ J. C. Slonczewski, J. Magn. Magn. Mater. 159, L1 (1996).

${ }^{10}$ D. A. Garanin, Phys. Rev. B 55, 3050 (1997).

${ }^{11}$ N. Kazantseva, D. Hinzke, U. Nowak, R. W. Chantrell, U. Atxitia, and O. Chubykalo-Fesenko, Phys. Rev. B 77, 184428 (2008).

${ }^{12}$ J. Kötzler, D. A. Garanin, M. Hartl, and L. Jahn, Phys. Rev. Lett. 71, 177 (1993).

${ }^{13}$ N. Kazantseva, R. Wieser, and U. Nowak, Phys. Rev. Lett. 94, 037206 (2005).

${ }^{14}$ D. Hinzke, N. Kazantseva, U. Nowak, O. N. Mryasov, P. Asselin, and R. W. Chantrell, Phys. Rev. B 77, 094407 (2008).
Walker threshold assuming the Gilbert form of damping or the LL form. Since the two behaviors can be qualitatively different, a measurement of the temperature dependence of the velocity and the Walker threshold could pose a unique opportunity to identify, whether the Gilbert or the LL formulation are the physically relevant one. This then in turn could have implications for determining the physically relevant description of damping, which is one of the key open questions in magnetization dynamics.

\section{ACKNOWLEDGMENTS}

The authors thank N. Kazantseva for helpful discussions. This work was funded by the Deutsche Forschungsgemeinschaft (Grant No. SFB 767), Landesstiftung BadenWürttemberg, and the ERC (Grant No. 2007-Stg 208162). Granting of computer time from HLRS, and NIC is gratefully acknowledged.

${ }^{15}$ O. Chubykalo-Fesenko, U. Nowak, R. W. Chantrell, and D. Garanin, Phys. Rev. B 74, 094436 (2006).

${ }^{16}$ N. Kazantseva, D. Hinzke, R. W. Chantrell, and U. Nowak, EPL 86, 27006 (2009).

${ }^{17}$ Y. B. Bazaliy, B. A. Jones, and S. C. Zhang, Phys. Rev. B 57, R3213 (1998).

${ }^{18}$ A. Thiaville, Y. Nakatani, J. Miltat, and N. Vernier, J. Appl. Phys. 95, 7049 (2004).

${ }^{19}$ Z. Li and S. Zhang, Phys. Rev. B 70, 024417 (2004).

${ }^{20}$ J. He, Z. Li, and S. Zhang, Phys. Rev. B 73, 184408 (2006).

${ }^{21}$ P. M. Haney and M. D. Stiles, Phys. Rev. B 80, 094418 (2009).

${ }^{22}$ M. D. Stiles, W. M. Saslow, M. J. Donahue, and A. Zangwill, Phys. Rev. B 75, 214423 (2007).

${ }^{23}$ N. Smith, Phys. Rev. B 78, 216401 (2008).

${ }^{24}$ M. D. Stiles, W. M. Saslow, M. J. Donahue, and A. Zangwill, Phys. Rev. B 78, 216402 (2008).

${ }^{25}$ N. L. Schryer and L. R. Walker, J. Appl. Phys. 45, 5406 (1974).

${ }^{26}$ J. L. García-Palacios and F. J. Lázaro, Phys. Rev. B 58, 14937 (1998).

${ }^{27}$ U. Nowak, in Annual Reviews of Computational Physics IX, edited by D. Stauffer (World Scientific, Singapore, 2001), p. 105.

${ }^{28}$ G. Tatara and H. Kohno, Phys. Rev. Lett. 92, 086601 (2004).

${ }^{29}$ C. Schieback, M. Kläui, U. Nowak, U. Rüdiger, and P. Nielaba, Eur. Phys. J. B 59, 429 (2007).

${ }^{30}$ R. A. Duine, A. S. Núñez, and A. H. MacDonald, Phys. Rev. Lett. 98, 056605 (2007).

${ }^{31}$ J. Xiao, A. Zangwill, and M. D. Stiles, Phys. Rev. B 73, 054428 (2006). 